\title{
To clip or not to clip? Noseclips for spirometry
}

\author{
R. Chavasse*,\#, P. Johnson" ${ }^{\#}$ J. Francis", I. Balfour-Lynn", M. Rosenthal", A. Bush"
}

To clip or not to clip? Noseclips for spirometry. R. Chavasse, P. Johnson, J. Francis, I. Balfour-Lynn, M. Rosenthal, A. Bush. C) ERS Journals Ltd 2003.

ABSTRACT: The use of noseclips for open-circuit spirometry is sporadic, despite guidelines encouraging their use. The authors aimed to evaluate whether noseclips significantly affected measurements of forced vital capacity (FVC) and forced expiratory volume in one second (FEV1) in children attending a tertiary, paediatric respiratory centre.

Children attending the asthma and cystic fibrosis (CF) clinics were asked to perform two sets of spirometry, one with and one without noseclips in random order, 20-min apart.

Paired data was obtained on 62 patients ( 32 asthma, $30 \mathrm{CF}$ ) with a median age of 11.4 yrs (range 7.2-17.2 yrs). There were no systematic differences in FEV1 or FVC measured with and without noseclips, although seven children $(11 \%)$ had clinically significant differences in FEV1 of $>190 \mathrm{~mL}$.

There is no clear advantage to wearing noseclips when performing open-circuit spirometry. Individuals should be assessed to ascertain their optimal technique, which should then be used consistently in clinical practice. Noseclips should probably be retained for research protocols.

Eur Respir J 2003; 21: 876-878.
*Queen Mary's Hospital for Children, Wrythe Lane, Carshalton, Surrey and \#Dept of Paediatric Respiratory Medicine, The Royal Brompton Hospital, Sydney Street, London, UK.

Correspondence: R. Chavasse, Queen Mary's Hospital for Children, Wrythe Lane, Carshalton, Surrey SM5 1AA, UK

Fax: 442086446878

E-mail: richard.chavasse@epsom-sthelier. nhs.uk

Keywords: Asthma

children

cystic fibrosis

noseclip

spirometry

Received: June 52002

Accepted after revision: January 62003
Open-circuit spirometry is frequently used in paediatric respiratory clinics to assess pulmonary status. Standardisation of technique is paramount to produce reproducible measurements of forced vital capacity (FVC), forced expiratory volume in one second (FEV1) and mean forced expiratory flows (FEF25-75\%). Guidelines for spirometry have been published by the American Thoracic Society (ATS) [1] and the European Respiratory Society [2] in which the use of noseclips is encouraged.

In a survey of 25 secondary and tertiary paediatric respiratory clinics in the $\mathrm{UK}$, noseclips were used routinely in only four $(16 \%)$, intermittently in one and not used in the remaining 20 . There is a perception that children may be frightened of, or dislike wearing noseclips. To the best of the authors' knowledge, the use of noseclips has not been assessed previously in children. They aimed to ascertain whether noseclips affected the measurements and reproducibility of FVC, FEV1 and FEF25-75\% in children with asthma and cystic fibrosis $(\mathrm{CF})$.

\section{Methods}

Subjects were recruited during attendance at the asthma and CF clinics at the authors' tertiary, paediatric respiratory centre. For inclusion, subjects had to have been diagnosed with either a diagnosis of asthma or $\mathrm{CF}$ and be aged between 6-18 yrs. To reduce variation related to operator technique rather than methodology, subjects had to have performed multiple previous tests (minimum of two). In practice, most subjects had performed numerous previous tests. Subjects were excluded if they had evidence of an acute illness or exacerbation of symptoms as this may also have increased variability. Patients attending the clinic do not normally wear noseclips.
Each subject was asked to perform two sets of spirometry, one with and one without noseclips. The sets were performed in random order, assigned by sealed envelope, stratified by disease. The two sets of measurements were performed on the same day with an interval of at least 20 min between sets. Each set consisted of three to five maximal expiratory flow volume manoeuvres to obtain three acceptable manoeuvres. Measurements were accepted in accordance with the ATS guidelines [1]. The best FVC and FEV1 were recorded for each set. FEF25-75\% was taken from the manoeuvre with the highest sum of FVC and FEV1 [1].

Following the second set of measurements, preference of technique was gauged by asking each subject "Did you find it easier to perform the test with or without noseclips?".

The mean \pm SD of FVC, FEV1 and FEF25-75\% were calculated for tests performed with and without noseclips. The difference in measurements from individual subjects was compared using the BLAND and ALTMAN [3] technique, giving mean difference \pm SD and $95 \%$ limits of agreement (mean difference \pm 2 SD of differences). A clinically significant difference between techniques was defined as an absolute difference in FEV1 of $190 \mathrm{~mL}$ [4]. The within-occasion variability of FEV1 and FVC was assessed by calculation of the coefficient of variation $(\mathrm{SD} / \mathrm{mean} \times 100)$ of the three manoeuvres in each set.

\section{Results}

Sixty-two subjects completed both tests of which 32 had asthma and 30 had CF. The median age was 11.4 yrs (range 7.2-17.2), and 29 were males (table 1). Two other studies were abandoned as the subjects became increasingly wheezy during testing (spirometry-induced bronchospasm). Six subjects left the clinic without performing the second set of measurements.

There was no systematic difference between measurements 
Table 1. - Subject characteristics

\begin{tabular}{lccc}
\hline & Asthma & CF & Total \\
\hline Subjects n & 32 & 30 & 62 \\
Age yrs & $11.5(7.2-16.9)$ & $11.0(7.7-17.2)$ & $11.4(7.2-17.2)$ \\
M:F & $16: 16$ & $13: 17$ & $29: 33$ \\
FVC L & $2.4 \pm 0.95$ & $2.13 \pm 0.77$ & $2.24 \pm 0.81$ \\
$\quad$ Mean \% pred & 92 & 88 & 89 \\
FEV1 L & $1.89 \pm 0.76$ & $1.63 \pm 0.59$ & $1.75 \pm 0.63$ \\
Mean \% pred & 78 & 73 & 75 \\
FEF25-75\% L·s & $1.79 \pm 0.78$ & $1.51 \pm 0.78$ & $1.65 \pm 0.78$ \\
Mean \% pred & 61 & 56 & 59 \\
\hline
\end{tabular}

Data are presented as median (range) or mean $\pm \mathrm{SD}$, unless otherwise stated. CF: cystic fibrosis; M: males; F: females; FVC: forced vital capacity; $\%$ pred: $\%$ predicted; FEV1: forced expiratory volume in one second; FEF25-75\%: mean forced expiratory flows. Baseline FVC and FEV1 performed without noseclips.

made with and without noseclips. The mean difference \pm SD in FEV1 (measurement with clips-measurement without clips) was $-15 \pm 138 \mathrm{~mL}$, limits of agreement $-291-261 \mathrm{~mL}$ (fig. 1). Seven subjects (11\%) of median age 12 yrs (11.2-15.2 yrs) had a difference in FEV1 of $\geqslant 190 \mathrm{~mL}$. In four, the higher value was obtained without using noseclips whilst in three it was greater with noseclips. The maximum absolute difference was $490 \mathrm{~mL}$ in one subject with CF. There was no relationship between the optimal technique and order of testing.

The mean difference \pm SD in FVC was $35 \pm 186 \mathrm{~mL}$, limits of agreement $-337-406 \mathrm{~mL}$. The mean difference \pm SD in FEF25-75\% was greater with a wider range $73 \pm 318 \mathrm{~mL} \cdot \mathrm{s}^{-1}$, limits of agreement $-706-560 \mathrm{~mL} \cdot \mathrm{s}^{-1}$.

Twenty-four subjects preferred performing spirometry without noseclips whilst 22 preferred using them. Sixteen subjects were unable to state a preference for either technique. The preferred method did not necessarily produce the best result nor was preference related to subject age.

The coefficient of variation of measurements of FEV1 was significantly lower when using noseclips $(2.94 \pm 2.11$ versus $4.45 \pm 3.4 \% ; \mathrm{p}<0.01)$ but there was no difference in FVC. Subjects with CF showed greater variation between manoeuvres than those with asthma, both with and without noseclips (table 2).

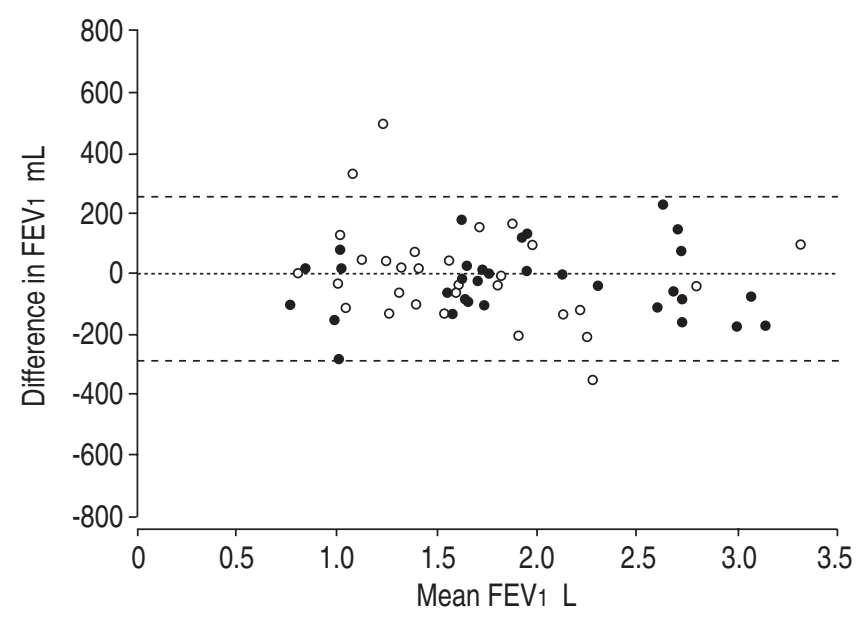

Fig. 1.-Bland-Altman plot of the difference in forced expiratory volume in one second $\left(\mathrm{FEV}_{1}\right)$ between clips and noseclips. $\bullet$ : asthma; $\bigcirc$ : cystic fibrosis; - - -: $95 \%$ limit of agreement.
Table 2. - Within-occasion coefficient of variation of forced expiratory volume in one second $\left(F E V_{1}\right)$ and forced vital capacity (FVC)

\begin{tabular}{lrc}
\hline & Asthma & CF \\
\hline FEV1 & & \\
$\quad$ With clips & $2.49(1.93)$ & $3.42(2.23)^{*}$ \\
$\quad$ Without clips & $3.7(3.35)$ & $5.24(3.34)^{*}$ \\
FVC & $3.22(2.13)$ & $3.85(2.65)$ \\
With clips & $3.92(4.04)$ & $4.74(3.11)$ \\
Without clips &
\end{tabular}

Data are presented as \% (SD). CF: cystic fibrosis. *: $\mathrm{p}<0.05$ clips versus no clips.

\section{Discussion}

The principle finding of this study is that the use of noseclips does not introduce any systematic bias in the measurement of FEV1, FVC or FEF25-75\%. There were individuals (seven of $62 ; 11 \%$ ) with clinically important differences between techniques (difference in FEV1 $\geqslant 190 \mathrm{~mL}$ ) but these differences occurred in both directions in similar numbers. To the best of the authors' knowledge there has been no similar study in children, however, similar results have been demonstrated in adults [5]. Surprisingly, there was no particular preference for or against using noseclips, and importantly, children did not appear to perform better with their preferred option.

Subjects who were accustomed to performing spirometry were studied in order to reduce excessive variability due to inexperience with the technique. Subjects with an exacerbation of symptoms were also excluded as increased variation may have occurred on repeated testing, potentially clouding the results. Only subjects with $\mathrm{CF}$ or asthma were studied. Results should only be extrapolated to subjects with alternative diagnoses undergoing pulmonary evaluation with care. Whilst subjects with, for example, idiopathic bronchiectasis would not be expected to differ from those with $\mathrm{CF}$, other patient groups may, such as those with neuromuscular conditions associated with palatial or facial weakness.

A weakness in this study is that it is cross-sectional. The authors have not shown whether findings in individuals are consistent over time (e.g. whether a child always performs better with or without noseclips). Furthermore, the authors have not been able to assess whether noseclips are beneficial to those performing the test infrequently or for the first time. Why some individuals performed better when either using noseclips or not cannot be explained.

The within-occasion variability in asthmatic children with asthma was less than in CF. Increased variability in CF has been demonstrated before [6] as has differences in variability between different spirometric indices (FEV1 versus FVC) $[6,7]$. Variability is decreased when wearing noseclips. This would reduce the magnitude of change required to detect a clinically significant event (e.g. bronchodilator response), if, as in many institutions, percentage change from baseline is used as the measure of significance.

Current reference ranges have, almost exclusively, been compiled using noseclips [8]. To make legitimate comparisons with these ranges, methodology should conform to that used in their construction. It would therefore be logical to encourage the use of noseclips. However, the present results would indicate that as a group, measurements made with and without noseclips are no different. Therefore, in clinical practice, it would be reasonable to compare measurements made without noseclips with the same reference range, supporting the practice of the majority of paediatric units in 
the UK. To maintain integrity, whilst using current reference ranges, research protocols should continue to include the use of noseclips.

In conclusion, the use of noseclips does not introduce any systematic bias in open-circuit spirometry. In view of the preference findings, it would seem reasonable to offer children a choice, recording what was actually used. In the absence of longitudinal data it would seem appropriate to be consistent in the technique used in subsequent measurements. If the pulmonary function results appear inconsistent with clinical state, it would be appropriate to repeat the tests using the opposite method. Comparisons between tests performed using the different techniques in individual patients should be made with caution.

\section{References}

1. American Thoracic Society. Standardisation of spirometry; 1994 update. Am J Respir Crit Care Med 1995; 152: 11071136.

2. Quanjer P, Tammeling G, Cotes J, et al. Lung volume and forced ventilatory flows; 1993 update. Working Party Report "Standardisation of lung function tests". European Coal and Steel Community and European Respiratory Society. Eur Respir J 1993; 6: Suppl. 16, 5-40.

3. Bland J, Altman D. Statistical methods for assessing agreement between two methods of clinical measurement. Lancet 1986; 1: 307-310.

4. Leading article. Reversibility of airflow obstruction: FEV1 vs. peak flow. Lancet 1992; 340: 85-86.

5. Yanev I. Importance of nasal clipping in screening investigations of flow volume curve. Folia Medica 1992; 34: 25-28.

6. Nickerson BG, Lemen RJ, Gerdes CB, Wegmann MJ, Robertson G. Within-subject variability and per cent change for significance of spirometry in normal subjects and in patients with cystic fibrosis. Am Rev Respir Dis 1980; 122: 859-866.

7. Strachan DP. Repeatability of ventilatory lung function measurements in a population survey of 7 year old children. Thorax 1989; 44: 474479.

8. Rosenthal M, Bain SH, Cramer D, et al. Lung function in white children aged 4-19 years: I - spirometry. Thorax 1993; 48: 794-802. 